\title{
Planar ESPAR Array Design with Nonsymmetrical Pattern by Means of Finite-Element Method, Domain Decomposition, and Spherical Wave Expansion
}

\author{
Jesús García, ${ }^{1}$ Juan F. Izquierdo, ${ }^{2}$ Jesús Rubio, ${ }^{2}$ Miguel A. González, ${ }^{1}$ and Juan Zapata ${ }^{1}$ \\ ${ }^{1}$ Departamento de Electromagnetismo y Teoría de Circuitos, Universidad Politécnica de Madrid, Ciudad Universitaria s/n, \\ 28040 Madrid, Spain \\ ${ }^{2}$ Departamento de Tecnología de los Computadores y de las Comunicaciones, \\ Universidad de Extremadura, Avenida de la Universidad s/n, 10003 Cáceres, Spain
}

Correspondence should be addressed to Juan F. Izquierdo, jfizquierdo@unex.es

Received 26 June 2012; Revised 2 August 2012; Accepted 16 August 2012

Academic Editor: Z. N. Chen

Copyright ( $\odot 2012$ Jesús García et al. This is an open access article distributed under the Creative Commons Attribution License, which permits unrestricted use, distribution, and reproduction in any medium, provided the original work is properly cited.

\begin{abstract}
The application of a 3D domain decomposition finite-element and spherical mode expansion for the design of planar ESPAR (electronically steerable passive array radiator) made with probe-fed circular microstrip patches is presented in this work. A global generalized scattering matrix (GSM) in terms of spherical modes is obtained analytically from the GSM of the isolated patches by using rotation and translation properties of spherical waves. The whole behaviour of the array is characterized including all the mutual coupling effects between its elements. This procedure has been firstly validated by analyzing an array of monopoles on a ground plane, and then it has been applied to synthesize a prescribed radiation pattern optimizing the reactive loads connected to the feeding ports of the array of circular patches by means of a genetic algorithm.
\end{abstract}

\section{Introduction}

In a large number of applications, such as WLAN, the radiating elements are installed on the ceiling of the room. In these cases, it is very useful to produce a toroidal beam with linearly polarized fields to prevent the interference coming from reflections on the walls and the floor of the room. A range of $\theta$ from $10^{\circ}$ to $65^{\circ}$ is suggested for the beam, while $\phi$-symmetrical fields are preserved [1]. Neighbouring users become another source of interference. A good solution to prevent this interference is to break the symmetry on the $\phi$ of the beam, by placing radiation nulls and/or maximums in the appropriate directions. Therefore, sometimes it is necessary to obtain non- $\phi$-symmetrical radiation patterns with a maximum beam for $\theta$ from $10^{\circ}$ to $65^{\circ}$.

There are several approaches to set up a nonsymmetrical radiation pattern. As a relevant example, the ESPAR electronically steerable passive array radiator concept is introduced in [2]. It consists of an array of monopoles over a metallic plane. One of these monopoles is placed in the centre of a circumference. The other monopoles are located uniformly spaced on the circumference. The central monopole is the active radiating element and the others are reactively loaded by means of PIN diodes. A low profile array made of equilateral triangular patches is presented in [3]. The radiation pattern is confirmed by loading the nonactive patches with reactive microstrip stubs.

Other numerical techniques have been employed to analyze the aforementioned arrays, such as the method of moments $(\mathrm{MOM})$ in $[1,3-5]$, the finite-element method (FEM) jointly with genetic algorithms (GA) in [2] or with the gradient algorithm working with patch elements [6]. However, in all these cases, the array is always studied as a whole block, that is, all the electromagnetic fields have to be recalculated for the whole structure in each step of any optimization algorithm since all the elements of the array are 


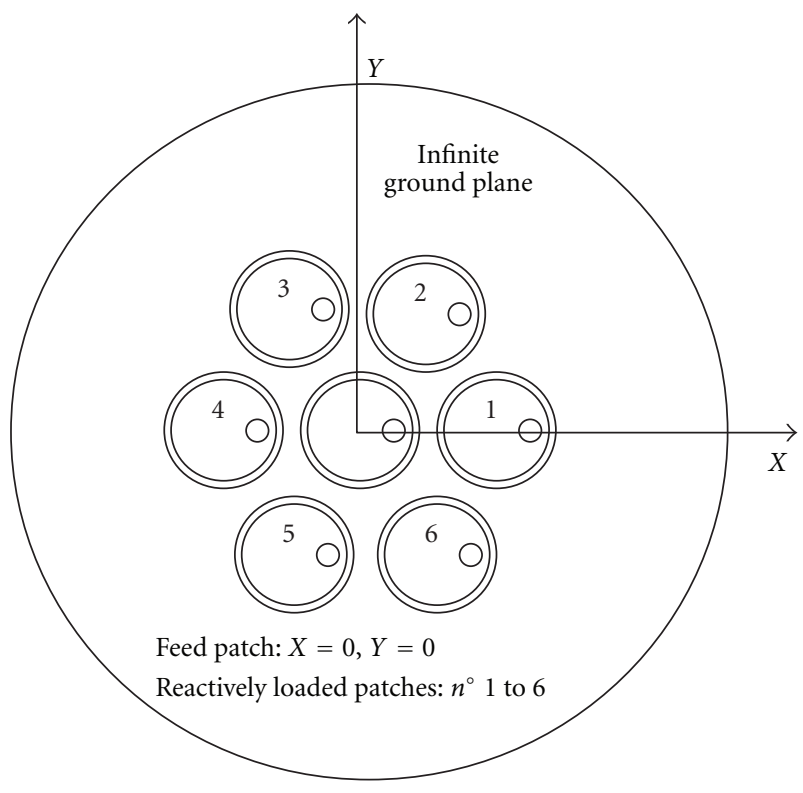

FIgURE 1: Planar ESPAR Array to be designed. Six reactively loaded patches over a infinite ground plane.

embedded in the same mesh. This makes the optimization a time-consuming task.

A method for the analysis of a class of arrays by using the 3D finite-element method (3D-FEM) and spherical mode expansion was presented in [7]. This technique is combined with a domain decomposition method, allowing the problem to be easily studied since the domain under study can be divided into several separate regions, thereby increasing the efficiency of the calculations. So, first the GSM of each antenna in terms of spherical modes and feed modes is obtained, and then the array is completely characterized by obtaining its overall GSM by means of an analytical procedure based on the translation theorems of spherical waves. Therefore, all the mutual coupling effects are taken into account since the scattering properties of each element can be calculated numerically and coupling and reflection characteristics of an array are easily obtained by using matrix expressions. The procedure provides coupling, scattering, and radiation properties of arrays, whose elements can be described in terms of spherical waves, rigorously. A reduced order model [8] may also be used to carry out a fast frequency sweep. The effectiveness of the proposed method for array design has been proved in other previous works $[9,10]$.

In order to validate the proposed procedure for this application, an array of monopoles will be analyzed and the results will be compared to the obtained in [4]. Finally, the method presented in [7] will be used to design and optimize a radiation pattern of an array of circular patches, see Figure 1, configured as an ESPAR array. Specifically, the patches will be fed by a coaxial probe, six of them will have reactive loads and only the central one will be fed. The optimization variables will be the reactive loads for all the nonactive patches.
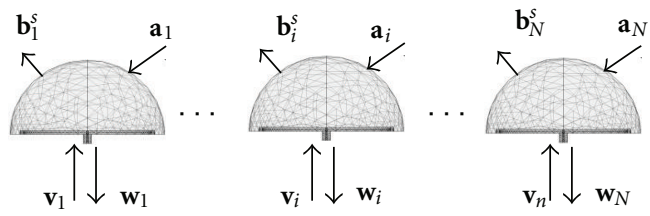

(a)

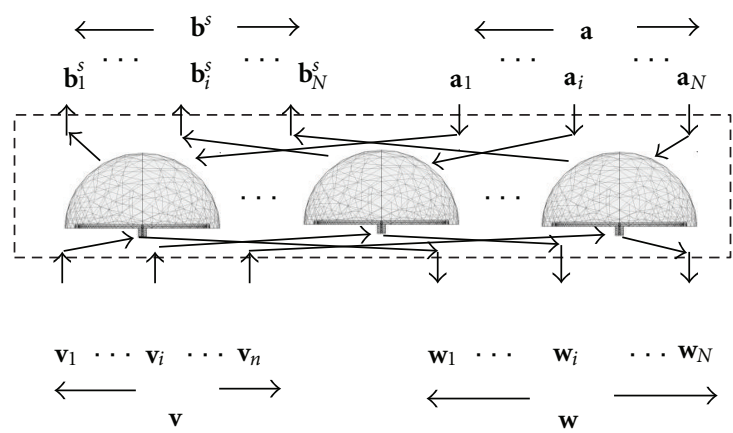

(b)

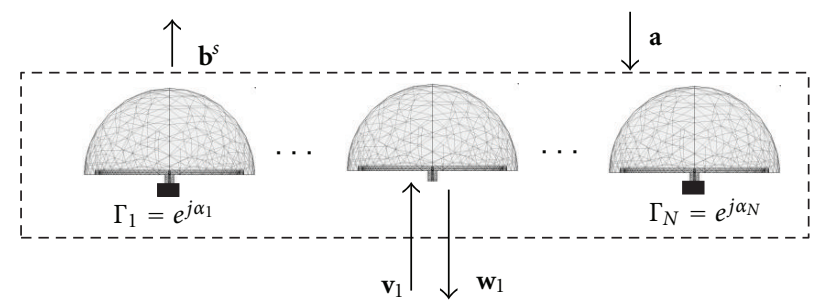

(c)

FIgUre 2: Computation of the GSM of the Planar ESPAR Array from the $\mathrm{GSM}_{i}$ of the isolated antennas.

\section{Design Procedure}

The design of the array will be performed as described in the following steps (Figure 2).

(1) Each one of the isolated elements of the array is completely surrounded by a hemisphere on an infinite ground plane, see Figure 2(a). This allows to describe each element in terms of spherical modes since the electromagnetic fields on the radiation port (hemisphere) can be expressed as a sum of spherical waves. All the elements of the array are identical, so only one GSM has to be numerically calculated. For an antenna with two ports, its $\mathrm{GSM}_{i}$ may be expressed as

$$
\left[\begin{array}{cc}
\boldsymbol{\rho}_{i} & \mathbf{r}_{i} \\
\mathbf{t}_{i} & \mathbf{s}_{i}-\mathbf{I}_{i}
\end{array}\right]\left[\begin{array}{l}
\mathbf{v}_{i} \\
\mathbf{a}_{i}
\end{array}\right]=\left[\begin{array}{l}
\mathbf{w}_{i} \\
\mathbf{b}_{i}^{s}
\end{array}\right]
$$

with $\mathbf{I}$ being the identity matrix, $\mathbf{b}^{s}$ are the complex amplitudes of the spherical modes in which the field scattered by the antenna is expanded. This may be expressed as $\mathbf{b}^{s}=\mathbf{b}-\mathbf{a}$ where $\mathbf{a}$ and $\mathbf{b}$ are the complex amplitudes of the incoming and outgoing spherical modes in the radiation port, $\mathbf{v}$ and $\mathbf{w}$ are the complex amplitudes of incident and reflected modes on the 
feed transmission lines or waveguides. All of them are column vectors. $\boldsymbol{\rho}_{i}, \mathbf{r}_{i}, \mathbf{t}_{i}$, and $\mathbf{s}_{i}$ are, respectively, the antenna reflection, reception, transmission, and scattering matrices.

(2) The overall multimode $S$-parameter matrix for the array is obtained from the $\mathrm{GSM}_{i}$, see Figure 2(b), by using the rotation and translation properties of spherical waves, accounting for all the mutual coupling effects between its elements. This way, it is possible to obtain the overall GSM for the array:

$$
\left[\begin{array}{cc}
\mathbf{\Gamma}_{G} & \mathbf{R}_{G} \\
\mathbf{T}_{G} & \left(\mathbf{S}_{G}-\mathbf{I}_{G}\right)
\end{array}\right]\left[\begin{array}{c}
\mathbf{v} \\
\mathbf{a}_{d}
\end{array}\right]=\left[\begin{array}{c}
\mathbf{w} \\
\mathbf{b}^{s}
\end{array}\right]
$$

where

$$
\begin{aligned}
\boldsymbol{\Gamma}_{G} & =\mathbf{\Gamma}+\mathbf{R G}[\mathbf{I}-(\mathbf{S}-\mathbf{I}) \mathbf{G}]^{-1} \mathbf{T}, \\
\mathbf{R}_{G} & =\mathbf{R}+\mathbf{R G}[\mathbf{I}-(\mathbf{S}-\mathbf{I}) \mathbf{G}]^{-1}(\mathbf{S}-\mathbf{I}), \\
\mathbf{T}_{G} & =[\mathbf{I}-(\mathbf{S}-\mathbf{I}) \mathbf{G}]^{-1} \mathbf{T}, \\
\left(\mathbf{S}_{G}-\mathbf{I}_{G}\right) & =[\mathbf{I}-(\mathbf{S}-\mathbf{I}) \mathbf{G}]^{-1}(\mathbf{S}-\mathbf{I})
\end{aligned}
$$

being

$$
\begin{gathered}
\mathbf{G}=\left[\begin{array}{ccccc}
\mathbf{0} & \mathbf{G}_{12} & \cdots & \cdots & \mathbf{G}_{1 N} \\
\mathbf{G}_{21} & \mathbf{0} & \ddots & \mathbf{G}_{i j} & \vdots \\
\vdots & \ddots & \ddots & \ddots & \vdots \\
\vdots & \mathbf{G}_{j i} & \ddots & \mathbf{0} & \mathbf{G}_{N-1 N} \\
\mathbf{G}_{N 1} & \cdots & \cdots & \mathbf{G}_{N N-1} & \mathbf{0}
\end{array}\right], \\
\mathbf{v}=\left\{\begin{array}{c}
\mathbf{v}_{1} \\
\vdots \\
\mathbf{v}_{i} \\
\vdots \\
\mathbf{w}_{N}
\end{array}\right\}, \quad \mathbf{w}^{\prime}=\left\{\begin{array}{c}
\mathbf{w}_{i} \\
\vdots \\
\mathbf{w}_{N}
\end{array}\right\} \\
\mathbf{T}=\operatorname{diag}\left(\mathbf{t}_{i}\right), \\
\mathbf{\Gamma}=\operatorname{diag}\left(\boldsymbol{\rho}_{i}\right), \quad \quad \mathbf{R}=\operatorname{diag}\left(\mathbf{r}_{i}\right), \\
\mathbf{b}_{1}^{s} \\
\vdots \\
\mathbf{a}_{d}=\left\{\begin{array}{c}
\mathbf{S}-\mathbf{I})=\operatorname{diag}\left(\mathbf{s}_{i}-\mathbf{I}_{i}\right) \\
\mathbf{a}_{d i} \\
\vdots \\
\mathbf{a}_{d N}
\end{array}\right\}, \quad \mathbf{b}^{s}=\left\{\begin{array}{c}
\mathbf{b}_{i}^{s} \\
\vdots \\
\mathbf{b}_{N}^{s}
\end{array}\right\}
\end{gathered}
$$

and the matrices $\mathbf{G}_{i j}$ contain information on the translation of spherical waves between elements $i$ and $j$ (general translations matrices). Details can be found in [7].

(3) The feed transmission lines corresponding to the nonactive antennas are loaded with pure reactances to obtain a desired radiation pattern, see Figure 2(c).
From the overall GSM of the array, this is a completely analytical step carried out through the reflection coefficient of the load, $\mathbf{v}$ and $\mathbf{w}$. In this step, the variables are the phases of the reflection coefficients presented by the loads.

(4) The radiation pattern is obtained by assuming $\mathbf{a}_{\mathbf{d}}=\mathbf{0}$ (non incident field on the array) as

$$
\mathbf{E}_{G}(\rho, \varphi, \theta)=\sum_{i=1}^{N}\left(\mathbf{b}_{i}^{s} \cdot \mathbf{e}_{i}\right) e^{j k \sin \theta\left(x_{i} \cos \varphi+y_{i} \sin \varphi\right)} .
$$

(5) The reactive loads on the feeding ports are changed.

(6) Go to step 3 until the synthesized radiation pattern obtained fulfils the prescribed design specifications.

It is worth mentioning that the main numerical effort in this procedure is made just once, at the first step, and it is not necessary to repeat it. The second to fourth steps are fully analytical, which allows their efficient inclusion inside an optimization procedure to obtain the desired radiation pattern.

\section{Validation}

In order to validate the proposed procedure, it has been applied to study a structure based on monopoles and the results have been compared with those available in the references. Then, this procedure has also been applied to design an array of probe-fed circular microstrip patches, and a radiation pattern has been synthesized by means of a genetic algorithm.

So the first example considers an array of monopoles. This array is also studied in [4]. Its structure can be seen in Figures 3 and 4 of [4]. A central active monopole $15 \mathrm{~mm}$ long with diameter $1.5 \mathrm{~mm}$ is fed by a $50 \Omega$ coaxial probe, with air as the dielectric and a central conductor with diameter $1.5 \mathrm{~mm}$. This monopole is surrounded by eight monopoles of the same length and diameter uniformly distributed over a circumference of radius $6 \mathrm{~cm}$. Five of them are grounded and the other three are loaded with a $50 \Omega$ coaxial line, air as dielectric, central conductor of $1.5 \mathrm{~mm}$ diameter, with length $10 \mathrm{~mm}$ loaded with a short circuit. The array has been constructed over a large metallic ground plane.

In this work, we have firstly studied the array as a whole, thus obtaining the response of the array by embedding the whole array in the same hemispherical mesh. Its GSM has been calculated by using a radiating hemispherical port and 4 coaxial feeding lines. Then, the loads (short circuits through a length of coaxial line in this case) have been applied to the three corresponding monopoles, resulting in a device with one radiating spherical port and a coaxial line port. A comparison of our calculated results and the measurement and computed results given in [4] is shown in Figure 3(a) for the reflection coefficient and for the azimuthal radiation pattern in Figure 3(b), at a frequency of $5 \mathrm{GHz}$. It can be observed that the procedure for calculating the radiation properties of an array of antennas, considering it as a whole structure, is working properly. 


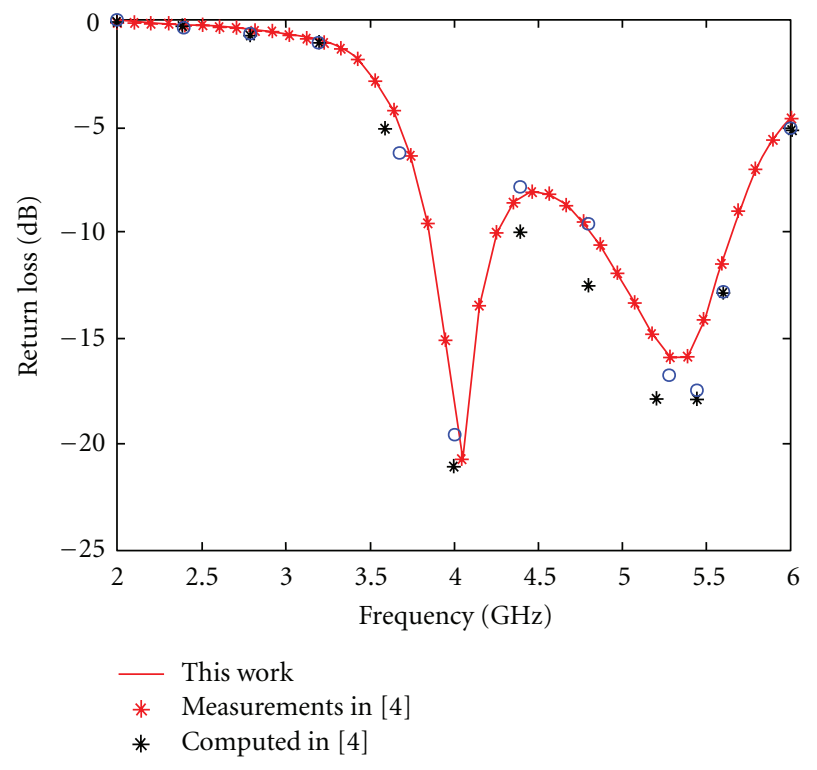

(a)

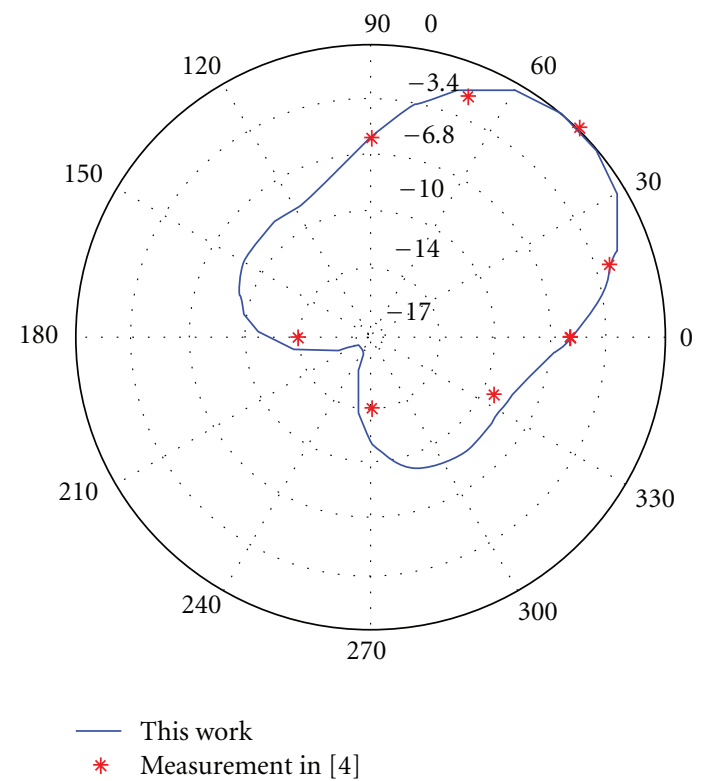

(b)

Figure 3: (a) Reflection coefficient; (b) the azimuthal radiation pattern; both at $5 \mathrm{GHz}$.

Next, we have slightly modified the previous monopole array. Now the nonradiating elements are distributed over a circumference of radius $10.5 \mathrm{~cm}$. All the other dimensions remain unchanged. This array has been studied twice. First, the array has been analyzed as a block and the results have been used for comparison purposes. Second, the array has been studied following the procedure described in Section 2, obtaining the overall GSM from the GSM of the isolated elements.

For the first case, where the array has been analyzed as a block, the 3D radiation pattern obtained has been represented in Figure 4(a). For the second case, where the array has been studied from the GSM of each single radiating element, an isolated monopole has been studied with a hemispherical surface with radius $2 \mathrm{~cm}$, enough to embed the monopole. The array has an active element located at the centre, three passive elements are loaded with a pure reactive impedance of $91 j \Omega$, and the remaining are short circuited. Both conditions have been imposed by loading the ports of the overall GSM properly. The 3D radiation pattern of the array computed from the GSM of the isolated monopole is given in Figure 4(b). In order to clarify the comparison between these results, a $2 \mathrm{D}$ representation of the radiation pattern on the azimuthal plane for both cases is also given in Figure 4(c), where a very good agreement can be observed. Therefore, the proposed procedure to obtain the global GSM of a reactively loaded array starting from the GSM of each isolated element is working as expected.

It is interesting to compare the time required for the calculation in both cases. For the whole array, analyzed with
33,054 degrees of freedom, the time calculation for a frequency point is 112 seconds (standard PC $2.8 \mathrm{GHz}$ ). For the isolated monopole, analyzed with 11,797 degrees of freedom, the calculation time is 24 seconds. Building the overall GSM is a very fast analytical computation (rotation and translation of spherical waves between the positions of the monopoles). Moreover, if a new distribution on the loading impedances and/or a new position of monopoles is required, the computing time is very low once the GSM of the isolated monopole has been calculated. This is the main advantage of the proposed procedure.

\section{Planar ESPAR Array Design}

The design procedure described above has also been applied to the design of a planar ESPAR of probe-fed circular microstrip patch antennas, with six identical elements as given in Figure 1. The design frequency is $2.41 \mathrm{GHz}$.

A single radiating element, a circular patch with a coaxial probe, has been designed. The patch is located over a dielectric disk of thickness $0.2 \mathrm{~cm}$, relative permittivity $\varepsilon_{r}=$ 1.07 , loss tangent $\operatorname{tg} \delta=0.0008$. The dimensions are radius of the dielectric disk $3.5 \mathrm{~cm}$, radius of the patch $3.32 \mathrm{~cm}$, zero thickness, and probe position $x p=1.0 \mathrm{~cm}$. Thus, a $-20 \mathrm{~dB}$ input reflection coefficient has been found at $2.41 \mathrm{GHz}$. The radiating structure, patch and dielectric disk, has been embedded in a hemisphere of radius $4 \mathrm{~cm}$. The calculation time for a frequency point is less than 2 minutes $(26,121$ degrees of freedom). 


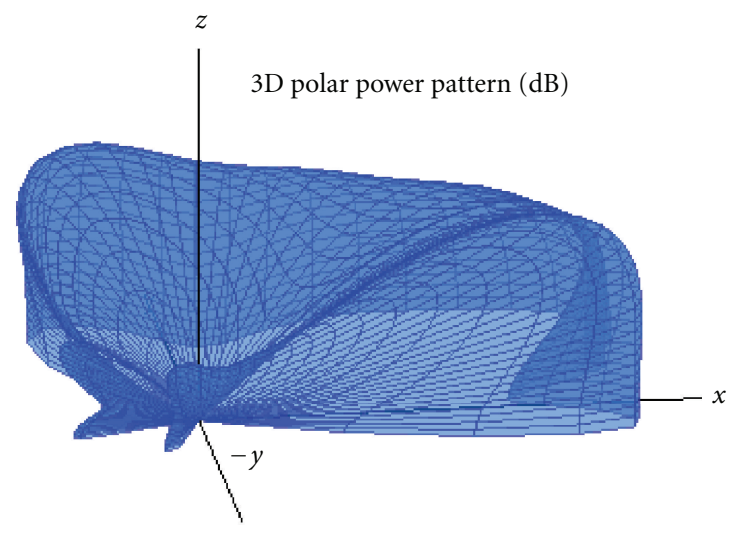

(a)

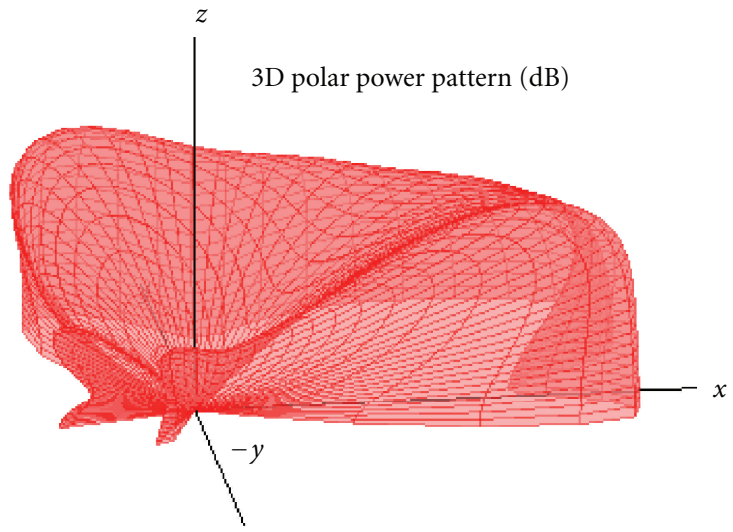

(b)

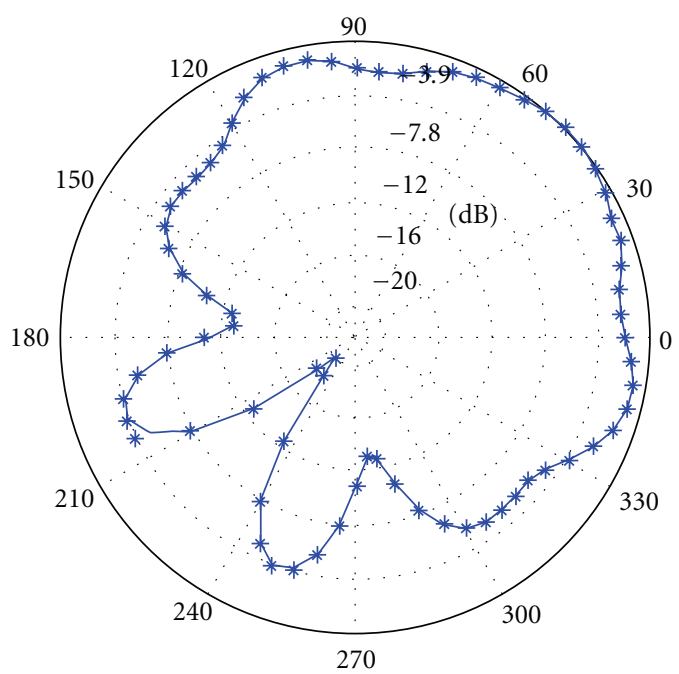

(c)

FIGURE 4: Results for a monopole array, radius $10.5 \mathrm{~cm}$, at $5 \mathrm{GHz}$. (a) 3D Polar representation of normalized power pattern calculated as a whole block. Values $10 \mathrm{~dB}$ below maximum are not represented. (b) 3D Polar representation of normalized power pattern calculated from the GSM of an isolated monopole. Values $10 \mathrm{~dB}$ below maximum are not represented. (c) Comparison of both cases on the azimuthal plane.

As stated before, once the GSM of the isolated circular patch is obtained, all the design is analytically calculated, with very low calculating times. A computation time smaller than 1 second may be considered for each one of the following radiation patterns.

The ESPAR array is made placing six identical reactively loaded circular patches over a circle of radius $R \mathrm{~cm}$, surrounding a central patch fed by a coaxial probe on an infinite ground plane. Several radiation patterns have been obtained by changing the reactance of each port with virtually no computational cost. The patches and the loads are hereinafter numbered as indicated in Figure 1.

\section{Radiation Pattern Optimization}

In order to obtain radiation patterns fulfilling some prescribed characteristics, a genetic algorithm has been applied to the design approach. In this case, the phases of the reflection coefficients for each one of the loading reactances have been chosen as the optimization variables. It should be noted that each optimization requires no more than 5 minutes.

It is remarkable that the proposed procedure allows the variation of the positions of the antennas without much effort by using the translational theorems of spherical waves, obtaining the full response of the array in a very short time. In this sense, the radius $R$ (containing the centre of the surrounding elements of the array) has been optimized to ensure a better performance of the design. This capability becomes a great advantage since there is no need to mesh again the whole array when the positions of the antennas have been changed due to a variation of the radius.

To illustrate the efficiency and the accuracy of the proposed optimization procedure, a radiation pattern with 


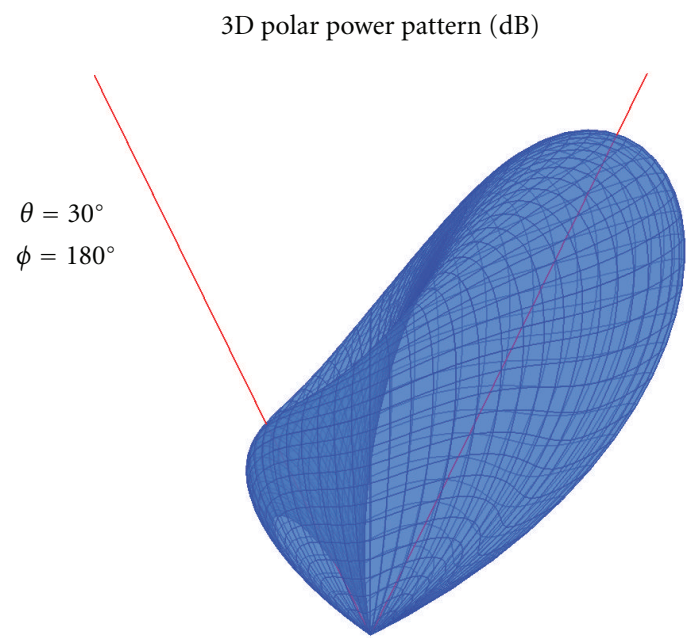

(a)

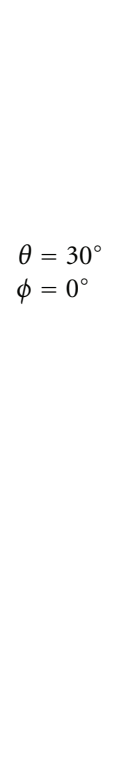

3D directivity (absolute value in $\mathrm{dB}$, CST MWS)
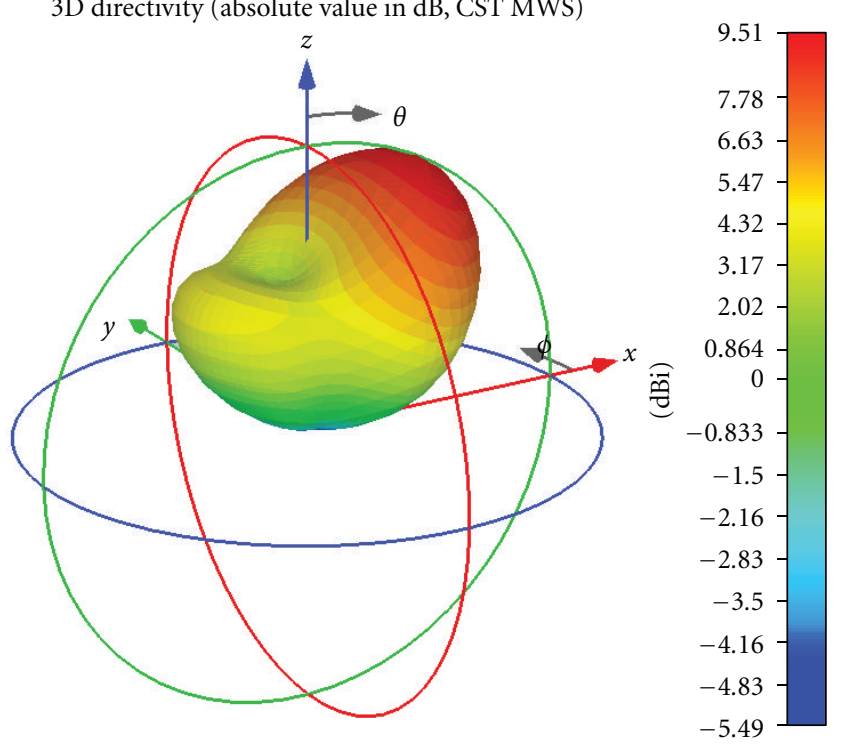

(b)

FIGURE 5: (a) 3D Polar normalized power pattern (values $20 \mathrm{~dB}$ below maximum are not represented) and (b) 3D Directivity pattern by means of CST MWS (absolute value in $\mathrm{dB}$ ), respectively, for an array with prescribed maximum at $\phi=0$ and $\theta=30^{\circ}$ and a attenuated value $7 \mathrm{~dB}$ below the maximum at $\phi=180$ and $\theta=30^{\circ}$. Loading reactances: $j X_{1}=9.52 j \Omega, j X_{2}=-42.55 j \Omega, j X_{3}=84.39 j \Omega, j X_{4}=242.69 j \Omega$, $X_{5}=66.39 j \Omega$, and $X_{6}=-45.83 j \Omega$. Both cases have been compared with the results obtained by CST Microwave Studio.

TABLE 1: Reflection coefficient at the operation frequency.

\begin{tabular}{lcc}
\hline & This work & CST MWS \\
\hline $\begin{array}{l}\text { Reflection coefficient } \\
\text { at } 2.41 \mathrm{GHz}\left(S_{11}\right)\end{array}$ & $-14.3143 \mathrm{~dB}$ & $-13.9777 \mathrm{~dB}$ \\
\hline
\end{tabular}

a maximum in $\phi=0$ and $\theta=30^{\circ}$ and a attenuated value $7 \mathrm{~dB}$ below the maximum in $\phi=180$ and $\theta=30^{\circ}$ has been specified in the design.

In this case, for a radius equal to $8 \mathrm{~cm}$, the reactances obtained from the optimization process have been found $j X_{1}=9.52 j \Omega, j X_{2}=-42.55 j \Omega, j X_{3}=84.39 j \Omega, j X_{4}=$ 242.69j $\Omega, X_{5}=66.39 j \Omega$, and $X_{6}=-45.83 j \Omega$. A 3D power pattern polar representation and a $3 \mathrm{D}$ directivity radiation pattern (absolute value in $\mathrm{dB}$ ) have been shown in Figure 5(a). For better understanding of the $3 \mathrm{D}$ representation, both directions (maximum and minimum) have been drawn as straight lines in Figure 5. Different cuts over the $\phi$-plane $(\phi$ $\left.=0^{\circ}, 30^{\circ}, 60^{\circ}, 90^{\circ}, 120^{\circ}, 150^{\circ}\right)$ for the copolar component of the radiation pattern have been represented in Figure 6. CST Microwave Studio software [11] has been used for validation purposes and a very good agreement has been observed in all cases.

Other radiation patterns subject to different specifications have also been synthesized with a good performance (not presented here to save space).

Additionally, we have studied the behaviour of the loaded structure with reactances from the point of view of the reflection coefficient at the operation frequency, with good agreement if compared to full-wave results obtained by means of CST MWS, as shown in Table 1.

\section{Conclusions}

The use of 3D FEM and spherical wave expansion becomes a very accurate method to analyze and design a circular patch planar ESPAR array. This method is also very efficient since most of the calculations can be carried out analytically. Obtaining the GSM of the isolated patch in terms of spherical waves is the only numerical computation required and, for this purpose, a hemisphere port surrounding the structure is considered. Once this GSM has been obtained, all the remaining calculations to achieve the GSM of the whole array, including the effects of the coupling between its elements, become very fast since they only involve the enforcement of theorems of rotation and translation of spherical waves, allowing its application to optimization procedures. Arrays with all their elements located very close to each other can be easily analyzed in order to obtain a high coupling factor between them. Also, the positions of the elements of an array can be easily and quickly changed avoiding the need to mesh again the whole structure.

A genetic algorithm optimization procedure has been used to synthesize a radiation pattern with some desired specifications in a short time. 


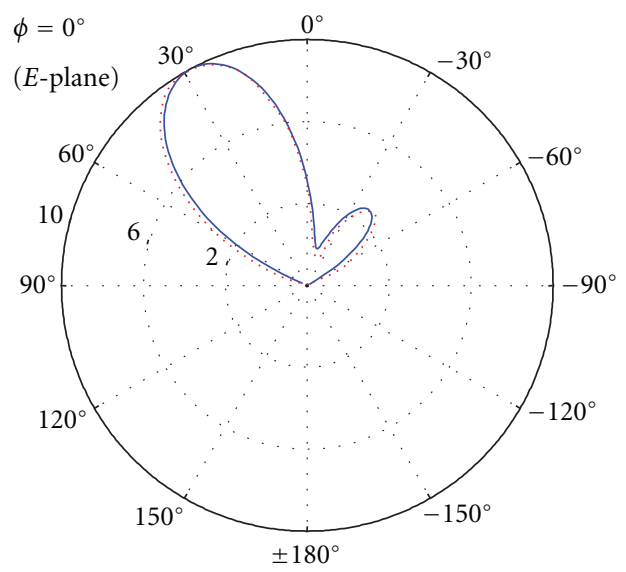

(a)

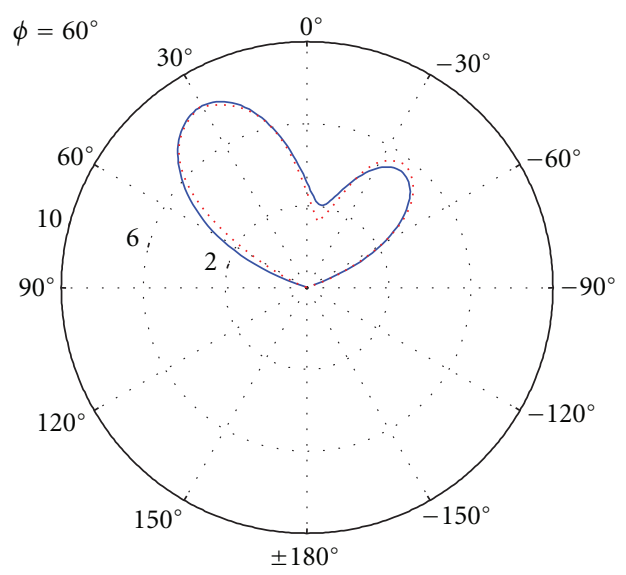

(c)

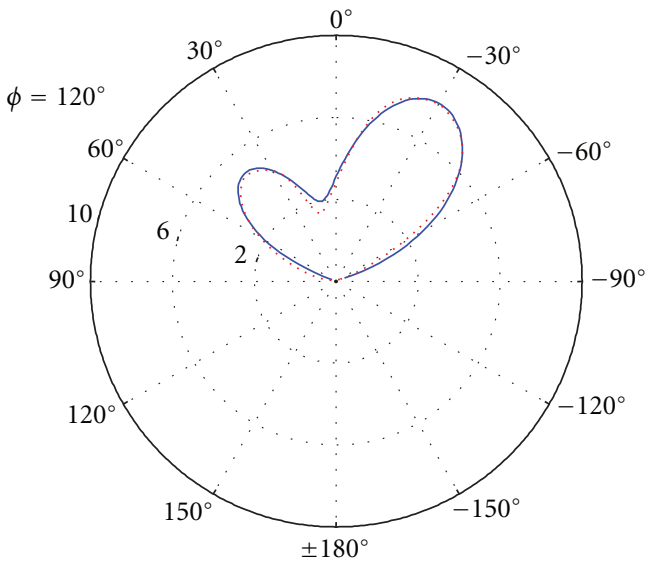

— This work

CST microwave studio

(e)

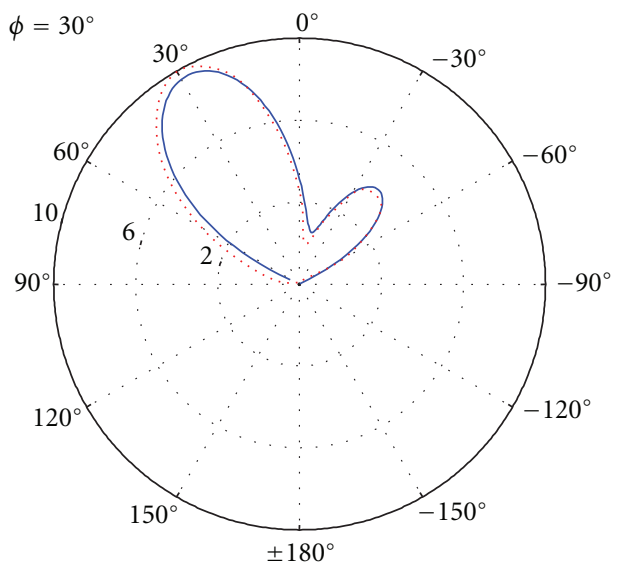

(b)

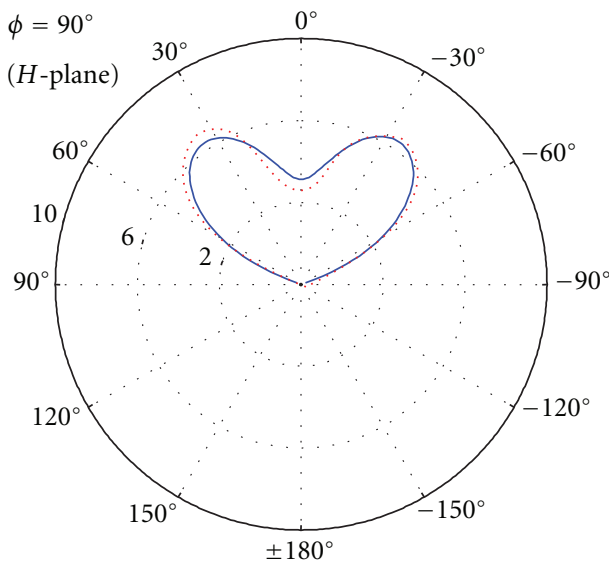

(d)

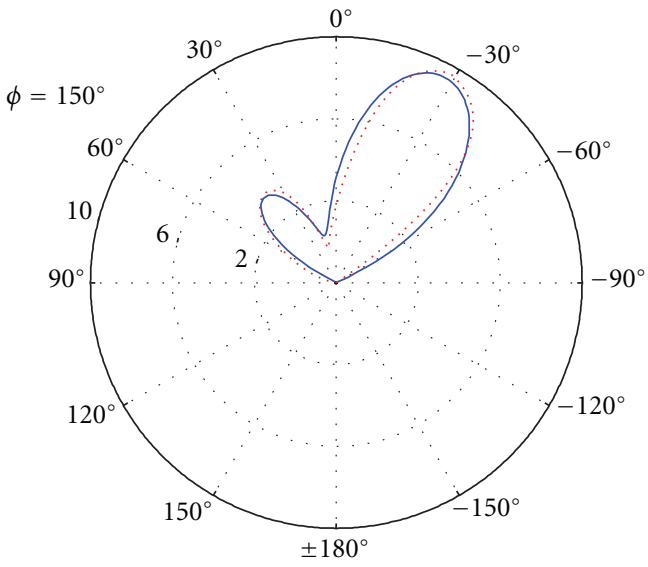

— This work

CST microwave studio

(f)

FIGURE 6: Different cuts over $\phi$-plane (copolar component of the radiation pattern) for an array with prescribed maximum at $\phi=0$ and $\theta=30^{\circ}$ and a attenuated value $7 \mathrm{~dB}$ below the maximum at $\phi=180$ and $\theta=30^{\circ}$. Loading reactances: $j X_{1}=9.52 j \Omega, j X_{2}=-42.55 j \Omega$, $j X_{3}=84.39 j \Omega, j X_{4}=242.69 j \Omega, X_{5}=66.39 j \Omega$, and $X_{6}=-45.83 j \Omega$. Full-wave results obtained by CST Microwave Studio have been included for comparison purposes. 


\section{Acknowledgments}

This work was supported by MICINN, Spain, under projects TEC2010-20249-C02-01, TEC2010-20249-C02-02, CSD2008-00068 Terasense and by FEDER and Junta de Extremadura under contract GR10126.

\section{References}

[1] J. C. Brégains, G. Franceschetti, A. G. Roederer, and F. Ares, "New toroidal beam antennas for WLAN communications," IEEE Transactions on Antennas and Propagation, vol. 55, no. 2, pp. 389-398, 2007.

[2] R. Schlub, J. Lu, and T. Ohira, "Seven-element ground skirt monopole ESPAR antenna design from a genetic algorithm and the finite element method," IEEE Transactions on Antennas and Propagation, vol. 51, no. 11, pp. 3033-3039, 2003.

[3] S. Fassetta and A. Sibille, "Low-profile circular array of equilateral triangular patches for angular diversity," IEE Proceedings, vol. 150, no. 1, pp. 34-36, 2003.

[4] A. Sibille, C. Roblin, and G. Poncelet, "Beam steering circular monopole arrays for wireless applications," in Proceedings of the 10th International Conference on Antennas and Propagation, Conference Publication no. 436, pp. 1.358-1.361, April 1997.

[5] W. H. Chen, J. W. Sun, X. Wang et al., "A novel planar switched parasitic array antenna with steered conical pattern," IEEE Transactions on Antennas and Propagation, vol. 55, no. 6, pp. 1883-1887, 2007.

[6] M. Shi, J. Lu, and D. J. Ireland, "Smart patch antenna for indoor mobile wireless computing," in Proceedings of AsiaPacific Microwave Conference (APMC '05), vol. 3, pp. 19131916, December 2005.

[7] J. Rubio, M. A. González, and J. Zapata, "Generalizedscattering-matrix analysis of a class of finite arrays of coupled antennas by using 3-D FEM and spherical mode expansion," IEEE Transactions on Antennas and Propagation, vol. 53, no. 3, pp. 1133-1144, 2005.

[8] J. Rubio, J. Arroyo, and J. Zapata, "SFELP—an efficient methodology for microwave circuit analysis," IEEE Transactions on Microwave Theory and Techniques, vol. 49, no. 3, pp. 509-516, 2001.

[9] J. Rubio, M. A. González, J. Zapata, A. Montesano, F. Monjas, and L. E. Cuesta, "Full-wave analysis of the Galileo system navigation antenna by means of the generalized scattering matrix of a finite array," in Proceedings of the 1st European Conference on Antennas and Propagation (EuCAP '06), Nice, France, November 2006.

[10] J. Corcoles, M. A. González, and J. Rubio, "Multiobjective optimization of real and coupled antenna array excitations via primal-dual, interior point filter method from spherical mode expansions," IEEE Transactions on Antennas and Propagation, vol. 57, no. 1, pp. 110-121, 2009.

[11] 3D EM Field Simulation-CST Computer Simulation Technology, http://www.cst.com. 

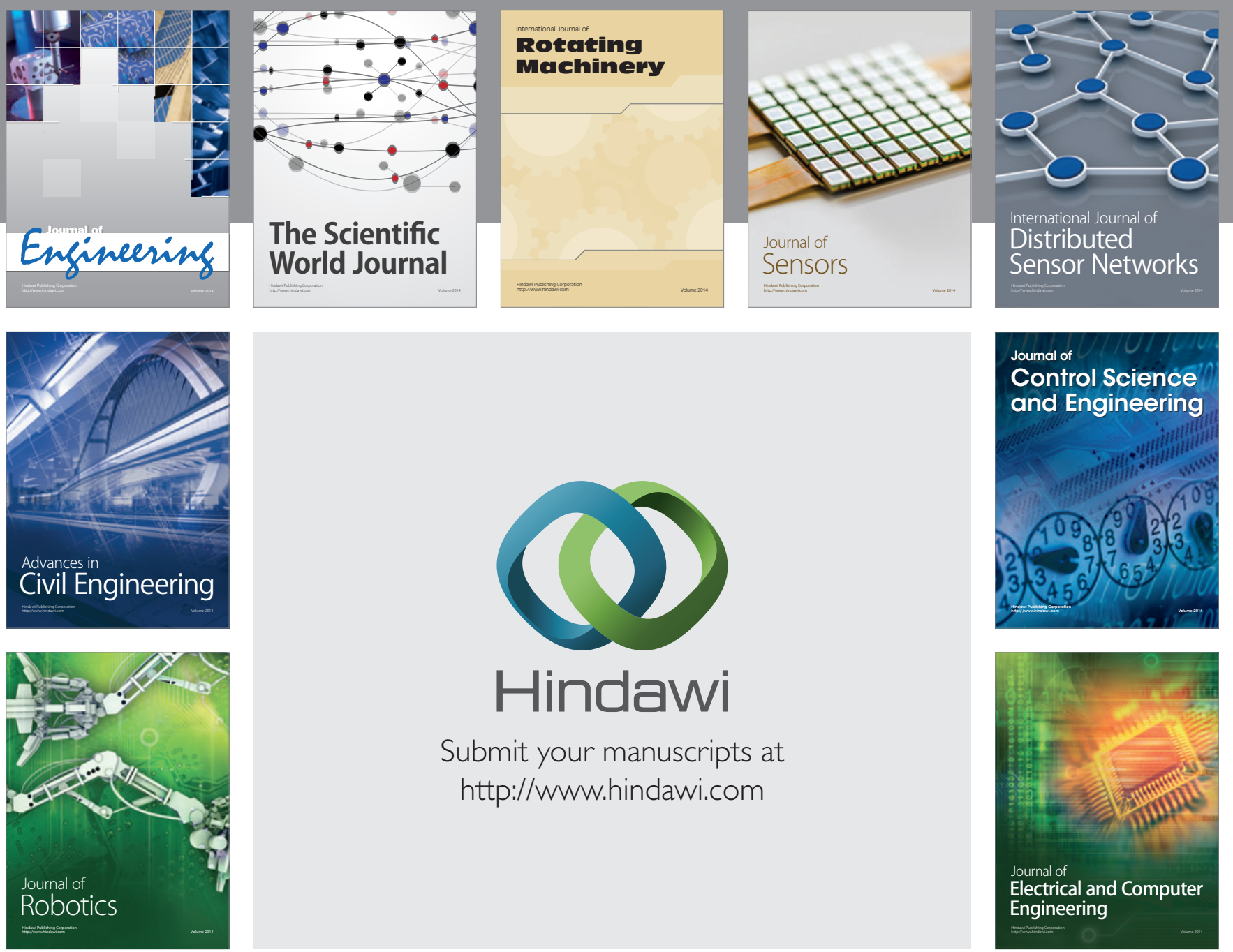

Submit your manuscripts at

http://www.hindawi.com
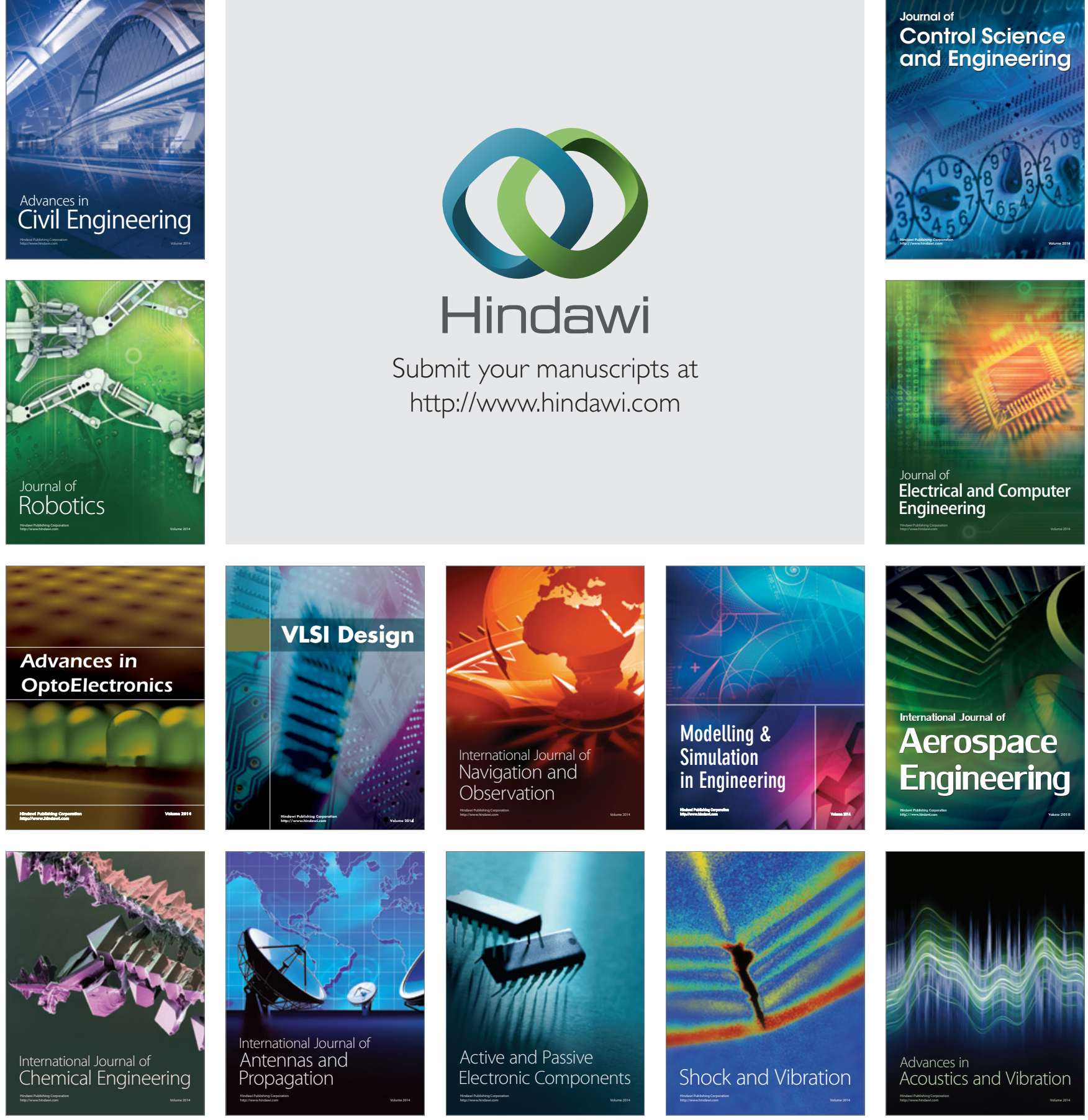